\title{
R-HyperCVAD/HD MTX AraC Regimen
}

National Cancer Institute

\section{Source}

National Cancer Institute. R-HyperCVAD/HD MTXAraC Regimen. NCI Thesaurus. Code C140115.

A regimen consisting of rituximab, cyclophosphamide, vincristine, doxorubicin and dexamethasone, administered on a hyperfractionated schedule, and alternating with rituximab, methotrexate and cytarabine that can be used for the treatment of mantle cell lymphoma (MCL) and acute lymphoblastic leukemia (ALL), as well as other types of lymphomas. 